\title{
Life Style Diseases
}

\author{
Dr. M. N Ramakrishna, M.D., A.F.I.H., PG.Dip. (Diab) \\ Medical officer, CVR College of Engineering \\ Email: drrkmn@ rediffmail.com
}

\begin{abstract}
Changes in social, cultural and economic status of people are resulting in people leaving the 'traditional life style' and adopting "modern westernized life style", thereby throwing them into new environment. As a result, they are suffering from some diseases called 'life style diseases', which can easily be prevented or postponed by adopting good life style. Examples of life style diseases are heart attacks (CAD), diabetes, obesity, certain cancers, liver disease, psychological problems etc. The article elaborates on the causes, the diseases and preventive steps.
\end{abstract}

Index Terms: Life Style Diseases, Hypertension, Diabetes, Atherosclerosis, Coronary artery disease, Asthma, Alcoholism, Abortion, Osteo-arthritis

\section{INTRODUCTION}

Life style diseases are a result of inappropriate relationship of people with their environments, which can potentially be prevented.

Globally 14.2 million people in the age groups of 30-69 years are subjected to premature death every year from these diseases.

The major causes of life style diseases include:

1. Drug abuse

2. Tobacco usage - smoking, chewing etc.

3. Alcohol abuse

4. Sedentary life \& lack of exercise

5. Dietary habits - like overeating, junk foods (which contain high fats) like pizza/burger, refined flours etc..

6. Stress

7. Sleeplessness

8. Adding pollution to the environment through excessive unnecessary usage of automobiles etc.

Some of the major diseases that can be included in these life style diseases are:

- Alzheimer's disease (loss of memory)

- Atherosclerosis (thickening of blood vessel walls)

- Hypertension (HTN)/ high blood pressure

- $\quad$ Type 2 diabetes (sugar disease)

- Coronary artery disease (heart attack)

- Cerebro-vascular accident/stroke(paralysis)

- Obesity

- Asthma

- Chronic pulmonary obstructive disease(COPD)

- Some cancers
- Chronic liver disease (cirrhosis of liver), chronic kidney disease(kidney failure)

- Depression

- Osteoporosis (weakening of bones due to loss of calcium)

- Road accidents, violence \& crime, family disorganization etc.

An estimate made by Harvard school of public health tells that the "Economic burden in India due to non-communicable diseases (which are a part of life style diseases) will be close to $\$ 6.2$ trillion for the period 2012 to 2030, which is equivalent to nearly 9 times the total health expenditure during the previous 19 years ( $\$ 710$ billions)".

The most important diseases -- Hypertension and Diabetes affect a person during productive years and can cause reduced productivity, and early retirement in addition to huge expenditure on health.

Information Technology (IT) sector plays predominant role in Indian "Economy" in terms of contribution to GDP and employment opportunity. Around $60 \%$ of young IT people are prone to life style diseases due to hectic work schedules, untimely and unhealthy eating habits, severe stress in the job, lack of exercise \& sedentary life style. Food items like pizza, noodles, burgers, fried foods, aerated drinks and alcohol (used to overcome stress) would contribute further.

As per some surveys conducted in corporate sector, it is revealed that

- $80 \%$ of corporate employees sleep less than 6 hours.

- $36 \%$ are obese

- $21 \%$ have depression

- $12 \%$ have high blood pressure at young age.

- $8 \%$ have diabetes at an early age.

\section{CAUSES OF LIFE STYLE DISEASES}

A).Drug Abuse:

People take drugs because they want a change in their lives.

Some reasons for young people getting addicted are:

- To fit in

- To escape/relax

- To relieve boredom

- To seem grownup 
- To rebel

- To experiment

They think drugs are a solution, but eventually the drugs become the problem. The consequences of drugs are always worse than the problems.

The drugs cause a false sense of euphoria, elation, hallucinations and lack of concentration, blurred memory and finally depression, leading to taking more quantity of same drug or shifting to different high potency drugs. They never come out of the vicious circle and ruin their career and lives.

Some of the drugs used for are:

- Marijuana(ganja)

- Alcohol

- Ecstacy

- LSD

- Amphetamine

- Heroin

- Morphine/pethidine

- Opium

- Some of the pain killers, tranquilizers, antidepressants, mood elevators etc., prescribed by doctors for various ailments, are also liable for addiction. Examples of such drugs are diazepam and alprazolam. They are sometimes used in high doses for committing suicide.

Drug abuse can cause dangerous diseases like HIV/AIDS, Hepatitis B, Hepatitis C infections (especially in intra-venous drug addicts), severe Psychosis, Depression, Suicidal tendencies, rejection in the society, loss of productive life, poor performance in studies and in work, family ruptures and also some chronic diseases like high BP, Heart ailments etc.

\section{B).Tobacco Usage:}

There is a saying that "some people commit suicide by 'drowning' but many by smoking". Usage of Tobacco by any means i.e. smoking cigarettes, beedi, chutta (cigar), chewing as powder, using in pans (jarada), snuff etc., is dangerous to health. The hazards of tobacco are innumerable. Some of them are:

a) Respiratory ailments like :

- Br. Asthma

- Chronic obstructive pulmonary disease (COPD)

- Chronic Bronchitis

- Reduced lung capacity (Vital capacity)

- Cancer Cheek

- Cancer Pharynx

- Cancer Larynx

- Cancer Hard Palate (Chutta Cancer)

- Cancer Lung etc... b) Gastro Intestinal Ailments include:

- Acute Gastritis

- Gastric and Duodenal ulcers

- Cancer oesophagus

- Cancer Stomach

- Cancer Pancreas

c) Cardio Vascular Ailments include:

- Heart Attack (CAD)

- Paralysis

- Periphenal-neurepathy

- Burger's Disease

- Heart Failure etc

d) Genito -Urinary Ailments include

- Cancer urinary bladder

- $\quad$ Impotence \& loss of libido

- Cancer Cervix in females.

e) Other body system ailments are

- $\quad$ Reduced vision/blindness

- Abortions/Low birth weight babies

- Early aging

- Wrinkled skin

- Decreased Stamina in sports.

- Decreased Productivity and loss of income due to health problems related to tobacco etc.

\section{C). Alcohol Abuse:}

Alcohol abuse is worldwide social and medical problem. Due to social, cultural and economic changes, people are getting addicted to alcohol at an early age. Some people view it as a symbol of prestige and social status. ailments:

Alcohol abuse can lead to the following

1. Acute/chronic gastritis

2. Gastric and duodenal ulcers

3. Rise in blood pressure which may precipitate heart attacks / cerebro-vascular accident (paralysis)

4. Chronic liver disease (Cirrhosis of liver) and liver cancer.

5. Acute pancreatitis

6. Cardio-myopathy

7. Peripheral neuropathy

8. Toxic psychosis

9. Cancer mouth, Pharynx, Larynx, Oesophagus etc

10. Automobile accidents (People using heavy machinery may meet accident under the influence of alcohol)

11. Injuries and death due to violence and crime

12. Suicidal tendency

13. Loss of productivity to organization and personal loss of income 


\section{D).Sedentary life style and lack of exercise}

Now a days in this competitive world, from the child hood, children are forced to concentrate more \& more on studies and they are neither encouraged in schools nor at home on games, sports, or some sort of physical exercise. Practically no physical exercise during the growing age and even after getting employment, because of hectic work schedule or odd timing of their jobs, and various easy methods of automobile transportation etc. lead to severe sedentary life, which in turn makes them overweight/obese.

Sedentary life style can lead to certain diseases like type II diabetes, high BP, Heart ailments, High fats in blood (hyper- cholesteremia), obesity etc.

Exercise improves blood circulation and regulates body weight and high fat in blood, prevents/postpones/controls high blood pressure, heart ailments, type II diabetes and increases good cholesterol in blood (high density lipid) which protects the heart, keeps the body in fit condition, and keeps away stress. As a consequence of this, concentration on studies / work is improved.

\section{E). Dietary Habits:} habits like

With or without knowledge change in dietary

1 Overeating(eating more than their requirement )

2 Under eating

3 Eating high calorie junk foods like pizza/burger etc

4 High intake of non-vegetarian food which contains high saturated fat (Meat, Chicken, Beef, Pork etc).

can cause:

- High blood pressure (Hypertension )

- Obesity

- Type II diabetes

- Protein calorie malnutrition

- Hyper cholesteremia (High fat in blood)

- Heart ailments

- Colon cancer (due to lack of dietary fiber)

- Stomach cancer(due to regular diet with smoked fish)

- Breast cancer (due to high fat diet)

F). Obesity:

$27 \%$ of urban population and $11 \%$ of rural population are obese. As per national family health survey (2005-06), 55 million people in India are obese. Obesity can lead to

- High blood pressure

- $\quad$ Type II diabetes

- Coronary heart disease (Heart attack)

- High fat in blood (hyper cholestremia)

- Gall bladder disease
- Breast cancer

- Varicose veins

- Abdominal hernia

- Psychological stress

- Lowered fertility

- Reduced life expectancy

- Early signs of osteo-arthritis in spine, hip, knee and ankle joint.

G).Stress:

Tensions due to family problems, financial problems, overwork burden, untimely working hours, sleeplessness etc can predispose stress in a person, and can lead to

- Drug abuse

- Alcoholism

- Smoking/tobacco usage

- Depression

- Precipitate/diabetes, high BP, heart problem etc.

- Ruptures in family relations etc.

What is the solution to get rid of these life style Diseases

Adoption of good life style is the solution. Some tips for better life style are :

1. Say no to drug abuse

2. Say no to alcohol

3. Say no to tobacco usage (smoking, chewing tobacco etc.)

4. Make regular exercise a part of life style

5. Check always your weight

6. Do not eat junk foods and adopt healthy dietary food habits, in the following way.

- $\quad$ Eat plenty of green leafy vegetables \& fruits

- Avoid fried foods and use vegetable oils in limited quantity (20 ml/head/day)

- $\quad$ Restrict eating chicken, mutton, beef, pork etc. as much as possible(because they contain bad fat)

- Fish can be liberally taken since it contains good fat.

- Add garlic to one food item as it reduces cholesterol and sugar, and prevents high BP and heart attacks

7. Cultivate the habit of regular meditation and yoga (age old practice of Pathanjali yoga), as they reduce stress.

8. Spare time for at least $6 \mathrm{hrs}$ sound sleep.

9. Corporate employers should see that working conditions of their employees should be properly monitored and potential of our youth should not be over exploited for getting more profit.

10. Fast food culture should be effectively controlled 
11. Automobile use should be restricted to bare minimum as a requirement for preventing pollution

12. Junior, Degree and Engineering colleges and Corporate IT companies should arrange for counseling sessions and awareness programmes at regular intervals on life style diseases

13. Corporate IT sector should arrange for medical check-ups at regular intervals, since early diagnosis can prevent and reduce life style diseases and their complications.

\section{CONCLUSION}

By taking minimum precautions and being conscious of the evils of inappropriate life style, it is possible to prevent many common health problems. It is necessary that the youth in particular be educated on the dangers of getting attracted to irregular and unhealthy habits in their lives. 